\title{
Opening again the debate: the transient nature of the circumnuclear disk
}

\author{
M. A. Requena-Torres ${ }^{1}$, E. A. C. Mills ${ }^{2}$, R. Güsten ${ }^{1}$, M. R. Morris ${ }^{3}$, \\ A. Weiss ${ }^{1}$, J. Martín-Pintado ${ }^{4}$, and A. Harris ${ }^{5}$ \\ ${ }^{1}$ Max-Planck-Institut für Radioastronomie, Germany, email: mrequena@mpifr.de; \\ ${ }^{2}$ NRAO Socorro, USA; ${ }^{3}$ University of California, Los Angeles, USA $;{ }^{4}$ CAB-INTA-CSIC, Spain; \\ ${ }^{5}$ Maryland University, USA
}

\begin{abstract}
Despite many investigations, the physical characteristics of the molecular gas in the Galactic center circumnuclear disk (CND) remain a topic of debate. Its mass is highly uncertain, between $10^{4}$ (from dust) and $10^{5-6} \mathrm{M}_{\odot}$ (derived from gas tracers), and depending on the probe, density estimates for the dense clumps are $10^{5-8} \mathrm{~cm}^{-3}$ and gas temperatures run from 50 to a few hundred K. The range of physical parameters leaves open many questions about the nature and fate of the CND. Using several ground-based observatories, together with Herschel and SOFIA, we have studied the physical conditions of the dense clumps using CO, HCN and $\mathrm{HCO}^{+}$, finding that most of them are transient. Their densities are not large enough for them to be gravitationally bound in the tidal field in the center of our Galaxy.
\end{abstract}

Keywords. Galactic center

\section{Introduction}

In the innermost region of the central molecular zone (CMZ) lies the last reservoir of molecular gas. Inside of this, UV radiation from the massive stars around the supermassive black hole in the center of our galaxy dissociate the molecules and only atomic and ionized gas share the place with the dust and stars that are observed in the infrared and optical regimes.

This disk-like structure extends from approximately 1.5 parsecs to 3 pc (Genzel et al. 2010) or $7 \mathrm{pc}$ depending on the source (Oka et al. 2011). The molecular CND seems to be the source of ionized gas streamers moving toward $\mathrm{Sgr} \mathrm{A}^{*}$ on highly eccentric orbits (although see the contribution by John Lacy, in this volume), forming the socalled minispiral. Martin et al. (2012) show that the CND is composed of at least three different rotating structures having different inclinations that fit with the arms of the minispiral.

The analysis of the excitation of the $\mathrm{CO}$ molecule, using low resolution far-infrared spectra obtained with the Kuiper Airborne Observatory (KAO), yielded high densities and high temperatures (Genzel et al. 1985; Harris et al. 1985; Bradford et al. 2005). But the inferred densities were not high enough to maintain clump stability against the strong tidal forces in the central gravitational potential. Those conclusions were questioned when interferometric data of the HCN $J=(1-0)$ and $J=(4-3)$ transitions from OVRO and SMA (Christopher et al. 2005; Montero-Castaño et al. 2009) were interpreted in terms of densities (assuming that the observed clumps are in fact viralized) large enough for clumps to be stable in those extreme conditions.

To further investigate the nature of the gas streams within the CND we have performed in-depth $\mathrm{CO}$ and $\mathrm{HCN} / \mathrm{HCO}^{+}$excitation studies. Complementing submm data from 

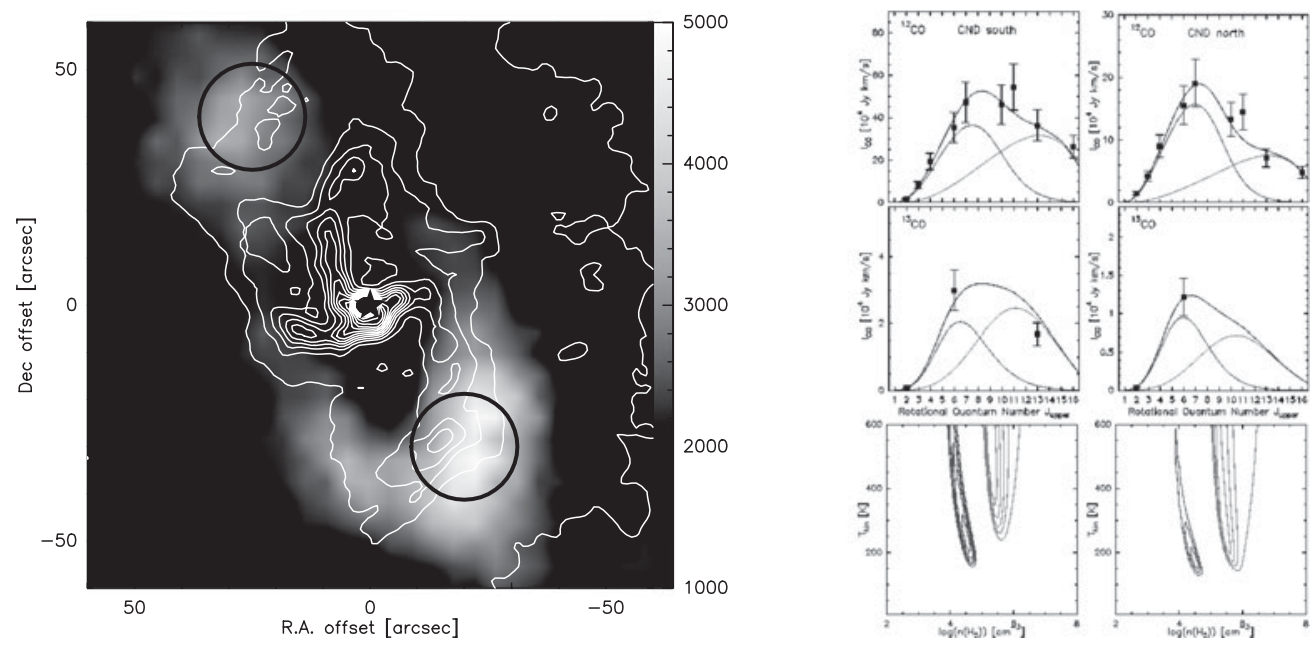

Figure 1. Left: Integrated emission of the CO $J=(6-5)$ line towards the CND, observed with the CHAMP + receiver at APEX (MR12). Right: Solutions of the SED fitting in the two main lobes of the CND shown as circles in the left figure (MR12).

APEX with airborne (SOFIA) and space (Herschel) observations, the new spectral energy distributions cover now a much wider range of excitation conditions. We present results of our CO (Requena-Torres et al. 2012, MR12), and of the $\mathrm{HCN}$ and $\mathrm{HCO}^{+}$excitation studies (Mills et al. 2013, M13) towards the two main lobes of the CND.

\section{Observations}

For the study of the conditions of the molecular material in the CND we have used a combination of millimeter and submillimeter telescopes.

Early observations mapped the $\mathrm{CO} J=(2-1)$ emission in the Sgr A complex with the IRAM 30-m telescope. Those were complemented by maps of the CO $J=(4-3)$ transition with the CSO, and more recently with all the submm transitions accessible to the APEX instruments (CO $J=(3-2), J=(4-3), J=(6-5)$, and $J=(7-6))$. With HIFI onboard the Herschel satellite we observed the CND region in the CO $J=(10-9)$ transition, and with SOFIA, the Stratospheric Observatory for IR Astronomy, we made use of the GREAT instrument to observe the $J=(11-10), J=(13-12)$, and $J=$ $(16-15)$ transitions in the two main lobes of the CND, where the CO $J=(7-6)$ emission peaks. We observed as well ${ }^{13} \mathrm{C}$ isotopologues with $\operatorname{APEX}(J=(3-2), J=(6-5))$ and SOFIA $(J=(13-12))$. As a parallel project, we mapped the CND in the high-J lines of $\mathrm{HCO}^{+}$and $\mathrm{HCN}$ accessible from $\operatorname{APEX}(J=(3-2), J=(4-3), J=(8-7)$, $J=(9-8))$, together with some of their isotopologues (see M13 for details).

\section{CO SED in the main lobes of the circumnuclear disk}

Using the CND maps of the CO transitions, from $J=(2-1)$ to $J=(16-15)$, we model the CO spectral energy distribution (SED) from the line resolved observations, convolving all the maps to the same beam size of $22.5^{\prime \prime}$ (the beam of the CO $J=(11-10)$ observations with GREAT). We used the ${ }^{13} \mathrm{C}$ isotopologues to constrain the optical depths of the CO lines. The non-LTE models used in the analysis are large velocity gradient models, similar to the ones described in Weiss et al. (2007). 

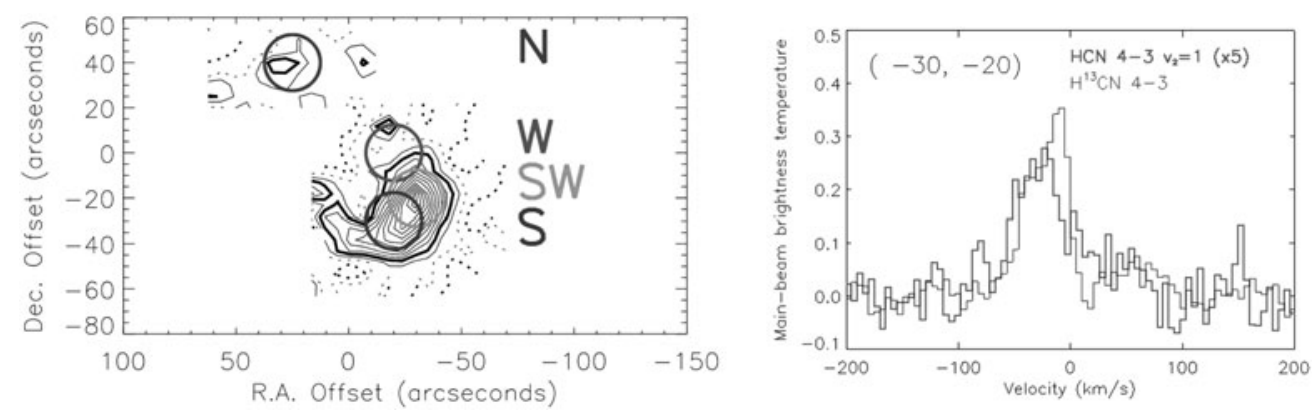

Figure 2. Left: Contour map of the $J=(8-7)$ transition of HCN observed with the APEX telescope. Circles show positions that have been studied (M13, their Figure 2). Right: Detection of the vibrationally excited $\mathrm{HCN}$ transition in the SW region, see M13.

The first result is that one needs two different excitation components of the gas to fit the CO SED. For both, we need high temperatures ranging from 175 to $500 \mathrm{~K}$, and densities from $10^{4}-10^{5} \mathrm{~cm}^{-3}$. The densities are tightly constrained in the reduced parameter space. The analysis implies that the gas cannot be gravitationally stable against tidal shear, as the densities are significantly below the Roche limit of $10^{7} \mathrm{~cm}^{-3}$.

\section{Excitation of the high density tracers: $\mathrm{HCN}$ and $\mathrm{HCO}^{+}$}

To study the conditions in the denser gas, we observed the high- $J \mathrm{HCN}$ and $\mathrm{HCO}^{+}$ lines observable with APEX (HCN $J=(3-2), J=(4-3)$, and $J=(8-7)$, as well as $\mathrm{HCO}+J=(3-2), J=(4-3)$, and $J=(9-8))$. Small maps were observed in all the transitions in order to study the two positions studied in $\mathrm{CO}(\mathrm{N}, \mathrm{S})$ and to extend the analysis to other two positions (W, SW, see M13).

Using the non-LTE radiative transfer code RADEX, the analysis was done in two ways, one using a wide velocity range for determining the average excitation, and the other probing individual clumps, assuming their emission is restricted to a relatively narrow velocity range $\left(20 \mathrm{~km} \mathrm{~s}^{-1}\right)$ based on the spectral profile of the peaks observed in HCN $J=(4-3)$ with interferometers (Montero-Castaño et al. 2009).

In both cases, all the solutions for the excitation of $\mathrm{HCN}$ and $\mathrm{HCO}^{+}$except one are consistent with those based on the CO (though on average, the densities are slightly higher), and indicate that densities are below the Roche limit. However the RADEX analysis of $\mathrm{HCN}$ emission in a small velocity range covering the so-called $\mathrm{S} 1$ source $\left(\mathrm{v}_{L S R}\right.$ : -115 to -95 , from $\mathrm{S}$ in Figure 2), shows a larger density that could hold a gravitationally bound clump in the CND.

But, toward one position in the CND (SW) we also detected the $J=(4-3) v_{2}=1$ vibrational excited line of $\mathrm{HCN}$, at a velocity of $-20 \mathrm{~km} \mathrm{~s}^{-1}$. This line is not likely to be excited by collisions as this requires densities of the order of $10^{11} \mathrm{~cm}^{-3}$, many orders of magnitude higher than any observed in the region. It is more likely that this line is excited by infrared pumping via rovibrational transitions, at $14 \mu \mathrm{m}$. Using a modified RATRAN code to model this pumping, we find that the most likely source of the excitation is warm ( $\mathrm{T} \sim 150 \mathrm{~K}$ ) dust embedded within the gas clump. This pumping also lowers the density inferred from the HCN excitation analysis. Due to the physical proximity of clumps S1 and SW, it is possible that $\mathrm{HCN}$ in $\mathrm{S} 1$ could also be subject to radiative excitation, which would lower its inferred density as well, potentially making it so this clump cannot be tidally stable. However, as the $v_{2}=1$ line is not detected at the velocity of clump S1, it is not clear how significant this effect may be. 


\section{Conclusions}

Using a combination of observations from ground-based telescopes, Herschel, and the GREAT instrument on-board SOFIA, we have obtained high-spatial and spectral resolution $\left(22.5^{\prime \prime}-8^{\prime \prime}\right.$ and $\sim 1 \mathrm{~km} \mathrm{~s}^{-1}$ ) $\mathrm{CO}$ maps of the circumnuclear disk (CND). The gas densities derived from our CO excitation analysis clearly imply that the CO-emitting clumps in the CND cannot be gravitationally stable against tidal shear, as they are well below the Roche limit of $10^{7} \mathrm{~cm}^{-3}$. As such, our models strongly suggest the CND consists of gravitationally-unbound, transient clumps.

An analysis of the denser gas component of the CND using multi-transition observations of $\mathrm{HCN}$ and $\mathrm{HCO}^{+}$from the APEX telescope also finds low densities $\left(10^{5}\right.$ $10^{6} \mathrm{~cm}^{-3}$ ) which supports the transient nature of the CND everywhere except in one position. In this specific position, the densities may be large enough to allow the material to be gravitationally bound. However, the discovery of vibrationally-excited HCN in a nearby clump indicates that warm embedded (T $150 \mathrm{~K})$ dust may contribute to radiative excitation of $\mathrm{HCN}$, and reduce the densities derived using this molecule.

Very recent works from Liu et al. (2013) and Smith \& Wardle (2013) using old and new interferometric data, also find that clumps in the CND are unlikely to be virialized, and agree that densities based on the assumption of virialization are overestimated. Upcoming observations with ALMA will allow for high resolution studies of the excitation of individual clumps, and should conclude this debate by determining if there truly are any individual clumps that are stable, or if the structure in the CND is entirely transient.

\section{References}

Brandford, C. M., Stacey, G. J., Nikola, T., et al. 2005, ApJ 623, 866

Christopher, M. H., Scoville, N. Z., Stolovy, S. R., et al. 2005, ApJ 622, 346

Harris, A. I., Jaffe, D. T., Silber, M., \& Genzel, R. 1985, ApJ 294, L93

Genzel, R., Watson, D. M., Crawford, M. K., \& Townes, C. H. 1985, ApJ 297, 766

Genzel, R., Eisenhauer, F., \& Gillessen, S. 2010, RvMP 82, 3121

Liu, H. B., Ho, P. T. P., Wright, M. C. H., et al. 2013, ApJ 770, L44

Martín, S., Martín-Pintado, J., Montero-Castaño, M., Ho, P. T. P., \& Blundell, R. 2012, A $\& A$ 539, A29

Montero-Castaño, M., Herrnstein, R. M., \& Ho, P. 2009, ApJ 695, 1477

Mills, E. A. C., Güsten, R., Requena-Torres, M. A., \& Morris, M. R. 2013, ApJ 779, 47

Oka, T., Nagai, M., Kamegai, K., \& Tanaka, K. 2011, ApJ 732, 120

Requena-Torres, M. A., Güsten, R., Weiss, et al. 2012, A $\& A$ 542, L21

Smith, I. L. \& Wardle, M. 2013, MNRAS, arXiv:1310.8429v1

Weiss, A., Downes, D., Neri, R., et al. 2007, A\&A 467, 955 